\title{
Cell motility affects the intensity of gap junctional coupling in prostate carcinoma and melanoma cell populations
}

\author{
ANNA DANIEL-WÓJCIK* ${ }^{*}$ KATARZYNA MISZTAL* , IGA BECHYNE, JOLANTA SROKA, \\ KATARZYNA MIEKUS, ZBIGNIEW MADEJA and JAROSLAW CZYZ \\ Department of Cell Biology, Faculty of Biochemistry, Biophysics and Biotechnology, \\ Jagiellonian University, ul. Gronostajowa 7, 30-387 Kraków, Poland \\ Received January 16, 2008; Accepted February 25, 2008
}

DOI: 10.3892/ijo_00000010

\begin{abstract}
Connexins and gap junctions play a crucial role during carcinogenesis. While diverse regulatory systems have been shown to modulate their function, the influence of cell motility on the intensity of gap junctional intercellular coupling has yet to be systematically addressed. Since cancer cell motility and intercellular coupling determine cancer development, we aimed at elucidating how mutual cell translocation modulates the intensity of gap junctional coupling in cell populations. Time-lapse analyses of the motility of connexin43 (Cx43)-coupled rat prostate carcinoma (MAT-LyLu) and mouse melanoma (B16) cells cultured on hyper-adhesive substrata revealed a reduced intensity of intercellular translocations in the two cell populations compared to the control conditions. While no detectable effects on the architecture of the actin cytoskeleton and Cx43 expression and phosphorylation were observed, the inhibition of cell motility was paralleled by an increase in the abundance of Cx43-positive plaques in cell-to-cell contacts and an enhancement of gap junctional coupling in cell populations cultured on hyper-adhesive substrata. Thus, a direct correlation between two cellular parameters crucial for carcinogenesis, i.e. cell motility and gap junctional coupling intensity exists in cancer cell populations.
\end{abstract}

\section{Introduction}

Gap junctions are aqueous intercellular channels formed by the docking of connexons (hexameres of connexin proteins), contributed by adjacent cells. In principle, these structures mediate the direct intercellular exchange of ions and small

Correspondence to: Dr Jaroslaw Czyz, Department of Cell Biology, Faculty of Biochemistry, Biophysics and Biotechnology, Jagiellonian University, ul. Gronostajowa 7, 30-387 Kraków, Poland

E-mail: jaro@mol.uj.edu.pl

${ }^{*}$ Contributed equally

Key words: carcinogenesis, cell motility, gap junctional coupling, connexin43, dye transfer metabolites, i.e. gap junctional intercellular coupling (GJIC), which takes part in the synchronization of cellular functions in tissues (1). The formation and function of gap junctional plaques is modulated by versatile systems regulating connexin expression, trafficking and turnover (2), channel conductivity (3) and cell adhesion $(4,5)$. The de-regulation of GJIC functions has been implicated in the development of a diverse array of diseases $(6)$, including tumorigenesis $(7,8)$. A release of neoplasic cells from a growth control imposed by the surrounding tissue was suggested to result from the impairment of GJIC (7), whereas coupling-independent interference of connexins with the function of regulatory pathways affecting the cell cycle has also been described (9-11). Similarly, the GJIC-independent facilitating effect of connexins on directed cell migration was described (12). These data indicate an existence of the network of interactions between major determinants of development and carcinogenesis, i.e. cell proliferation, motility and GJIC.

The active penetration of natural barriers such as the basement membrane and endothelial continuum by tumor cells is a crucial event during cell invasion and development of secondary tumors (13). Since the dynamics of cell-to-cell contacts was shown to play an important role in modulating GJIC $(4,5)$, cell migration, being a prerequisite of tumor cell invasion, may be of utmost importance in determining the intensity of GJIC. However, the effect of the magnitude of intercellular translocation in cell populations on the GJIC intensity has not yet been experimentally addressed.

We previously showed that rat prostate MAT-LyLu carcinoma cells are characterised by relatively high levels of Connexin43(Cx43)-mediated GJIC (14) and display relatively high 'contact-stimulated' motility, i.e. an induction of cell translocations is observed upon contact with neighbour cells (15). A similar phenomenon effect was observed in mouse melanoma B16 cell populations (16). This makes these cell lines a suitable model for elucidating the effect of the impairment of cell motility on GJIC. In the present study, the ability of poly-L-lysine (PLL)-coated substrata to affect mutual translocations in cell populations was exploited. PLL is a cationic polypeptide used in a wide spectrum of cell and molecular biology applications, such as gene transfer (17), the enhancement of biocompatibility of engraftments (18) and for the maintenance of poorly adhesive cells in an adherent state $(19,20)$. We demonstrated that the 
inhibition of cancer cell motility by hyper-adhesive, PLLcoated, substrata correlates with an increase in GJIC intensity without net effects on the actin cytoskeleton architecture, Cx43 expression and phosphorylation levels.

\section{Materials and methods}

Cell culture. Mouse melanoma B16 and rat prostate cancer MAT-LyLu (Dunning rat model) cells were cultivated in Minimal Essential Medium (MEM) and Roswell Park Memorial Institute-1640 (RPMI, Sigma) medium, respectively, supplemented as described $(15,16)$. For the analyses of the effect of poly-L-lysine (PLL)-coated substrata on cancer cell motility, Cx43 expression and GJIC, culture dishes (Corning) or slide glasses were pre-treated overnight with a pure boric buffer (control) or boric buffer containing 40 and $500 \mu \mathrm{g} / \mathrm{ml}$ of PLL (70-150 kDa; Sigma), rinsed with distilled water and air-dried (19). In each experiment, cells were plated on untreated and PLL-covered substrata at a density of 400 cells/ $\mathrm{mm}^{2}, 24 \mathrm{~h}$ prior to the experiment.

Time-lapse monitoring of the movement of individual cells. The movement of cells plated on untreated and PLL-covered substrata was time-lapse recorded for $4 \mathrm{~h}$ and the tracks of individual cells were determined from the series of changes in the cell centroid positions, pooled and analyzed as previously described $(14,21)$. This was performed in order to obtain: average speed of cell movement $(\mu \mathrm{m} / \mathrm{h})$, i.e. total length of cell trajectory/time of recording $(4 \mathrm{~h})$; total length of cell displacement $(\mu \mathrm{m})$, i.e. the distance from the starting point directly to the final position of the cell; and, in addition, average rate of cell displacement $(\mu \mathrm{m} / \mathrm{h})$, i.e. the distance from the starting point directly to the cell's final position/time of recording $(4 \mathrm{~h})$. Trajectories of cells from no less than three independent experiments (number of cells $>50$ ) were taken for the estimation of statistical significance by the nonparametric Mann-Whitney test.

Fluorescence microscopy analyses. For the analyses of the architecture of the actin cytoskeleton, cells were fixed with $3.7 \%$ formaldehyde, permeabilised with $0.1 \%$ Triton X-100, double-stained against vinculin (Sigma) and F-actin (phalloidin conjugated with TRITC; Sigma), labelled with Alexa 488-conjugated goat anti-mouse IgG (Molecular Probes) and visualised with a Leica DM IRE2 microscope. For immunocytochemical analyses of the intracellular localisation of $\mathrm{Cx} 43$, cells were fixed with methanol:acetone $\left(7: 3,-20^{\circ} \mathrm{C}\right)$, labelled with mouse anti-Cx43 IgM (Sigma), Cy3-conjugated goat anti-mouse IgM (Dianova) and counterstained with $0.5 \mu \mathrm{g} / \mathrm{ml}$ bis-benzimide (Hoechst) to visualise the nuclei (22). For each condition, the abundance of $\mathrm{Cx} 43$-positive plaques in at least three $(\mathrm{N}>3)$ independent specimens was semi-quantitatively assessed. The abundance of punctate Cx43-specific fluorescence was rated from (+) for immunoreactivity characteristic for controls, up to $(+++)$ for very high immunoreactivity.

Gap junctional intercellular coupling. GJIC was measured by a double fluorescent dye transfer assay as described (23). Briefly, donor cells labelled with calcein and DiI (Molecular
Probes) were plated onto monolayers of acceptor cells grown on glass control coverslips, or covered with PLL films as described above, at a ratio of 1:50 and incubated for $3 \mathrm{~h}$ at $37^{\circ} \mathrm{C}$. Afterwards, the coverslips were mounted on glass microscope slides and the intensity of GJIC was evaluated using a fluorescence Leica DM IRE2 microscope. Membrane intercalating DiI was used to visualize donor cells (red). Calcein (green), which is capable of diffusing to coupled neighbouring cells, is a marker of gap junctional coupling. The results are given as a coupling index $\left(c_{r}\right)$, i.e. the number of positive recipient cells per one donor cell. At least 50 donor cells per coverslip were analyzed in three independent experiments performed for each experimental condition (14).

Immunoblot analysis. Mat-LyLu and B16 cells were washed, harvested and dissolved in lysis buffer, and the protein content of lysates was determined as described (21). Cellular proteins (40 $\mu \mathrm{g} /$ lane) were applied to $12.5 \%$ SDS-polyacrylamide gels, followed by the transfer to nitrocellulose. Blots were exposed to primary (rb anti-Cx43) antibody and mouse anti-actin antibody (both from Sigma) followed by the detection of the antibodies using HRP-labeled secondary antibodies (Bio-Rad) and an ECL Western detection system (Pierce, Rockford, IL). Blots resulting from 3 independent Western blot assays were densitometrically assessed and analysed with a paired Student's t-test (14).

\section{Results}

Effect of PLL-coated substrata on the motility of MAT-LyLu and B16 cells. Time-lapse experiments revealed that the 'contact-stimulated' motility of MAT-LyLu and B16 cells $(15,16)$ was significantly inhibited on substrata covered with PLL compared to control conditions (Fig. 1). The value of the average speed of cell movement of MAT-LyLu cells decreased from $50.4 \mu \mathrm{m} / \mathrm{h}$ for control variants to $17.9 \mu \mathrm{m} / \mathrm{h}$, estimated for cells cultured on the substrata covered with $500 \mu \mathrm{g} / \mathrm{ml}$ poly-L-lysine (Fig. 1A-C, cf. D). This corresponded to an inhibition of MAT-LyLu cell displacement, i.e. a parameter directly affecting the stability of intercellular contacts (Fig. 1D, Table I). A corresponding inhibitory effect of PLL-coated substrata on cell movement and displacement rate was observed in melanoma B16 cell populations (Fig. 1E-G). This was illustrated by decreased values of the average speed of cell movement and the total length of B16 cell displacement, which decreased from $45.97 \mu \mathrm{m}$ in control conditions to $29.02 \mu \mathrm{m}$ estimated for cells cultured on substrata covered with $500 \mu \mathrm{g} /$ ml PLL (Fig. 1H, Table I). On the other hand, the observed inhibitory effect of PLL-coated substrata on the motility of cancer cells remained independent of the architecture of actin cytoskeleton. B16 cells formed prominent focal contacts (marked with arrows) visualised by the labeling of vinculin and F-actin (Fig. 1L), while these structures were absent in MAT-LyLu populations cultured in control conditions (Fig. 1I). A similar architecture of actin cytoskeleton and focal contacts was retained by analysed cells cultured on PLL-coated substrata (Fig. 1J and K cf. I; and M and N cf. L for MATLyLu and B16 cells, respectively). These data indicate that PLL affects cancer cell motility in a manner independent of the functional status of cell adhesive complexes. 
Control
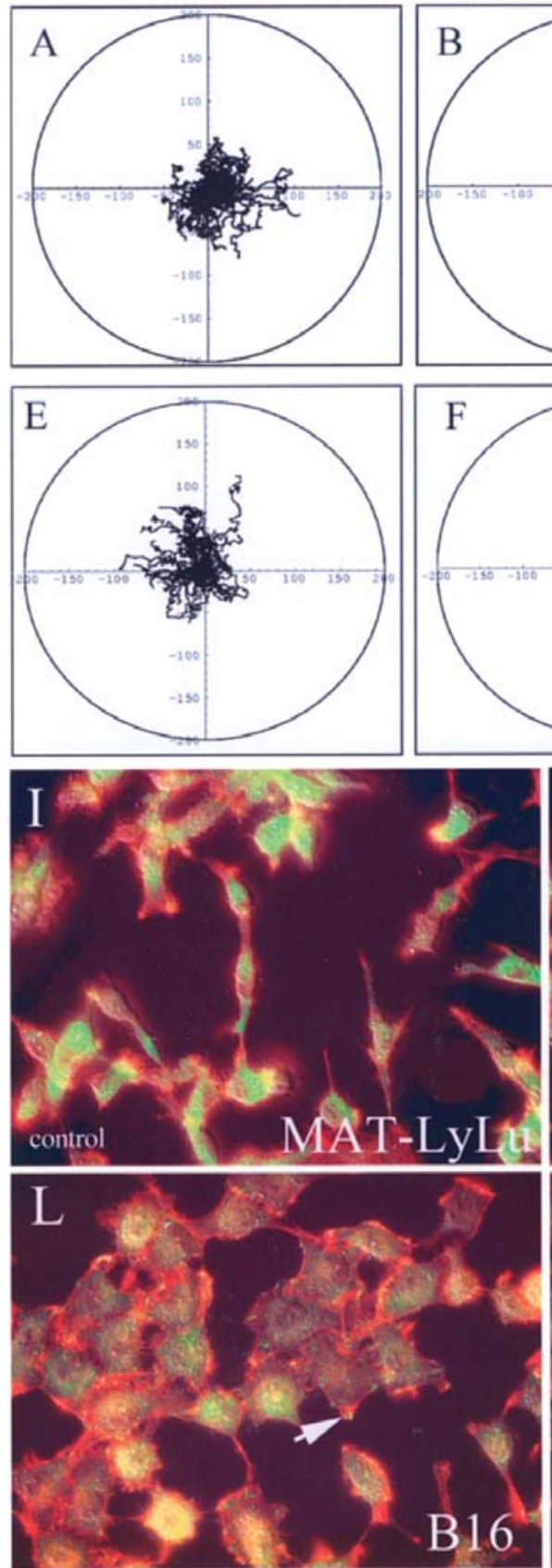

$40 \mu \mathrm{g} / \mathrm{ml}$ poly-L-lysine
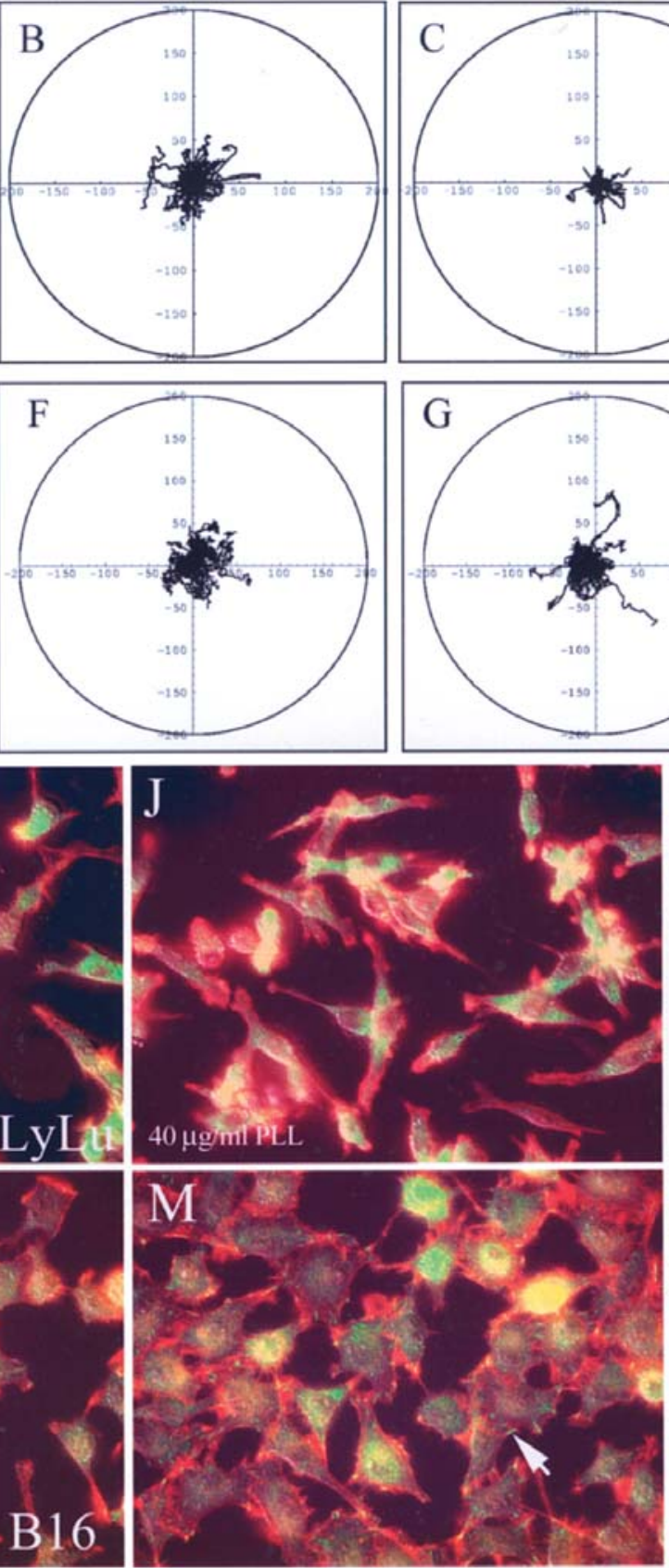

$500 \mu \mathrm{g} / \mathrm{ml}$ poly-L-lysine
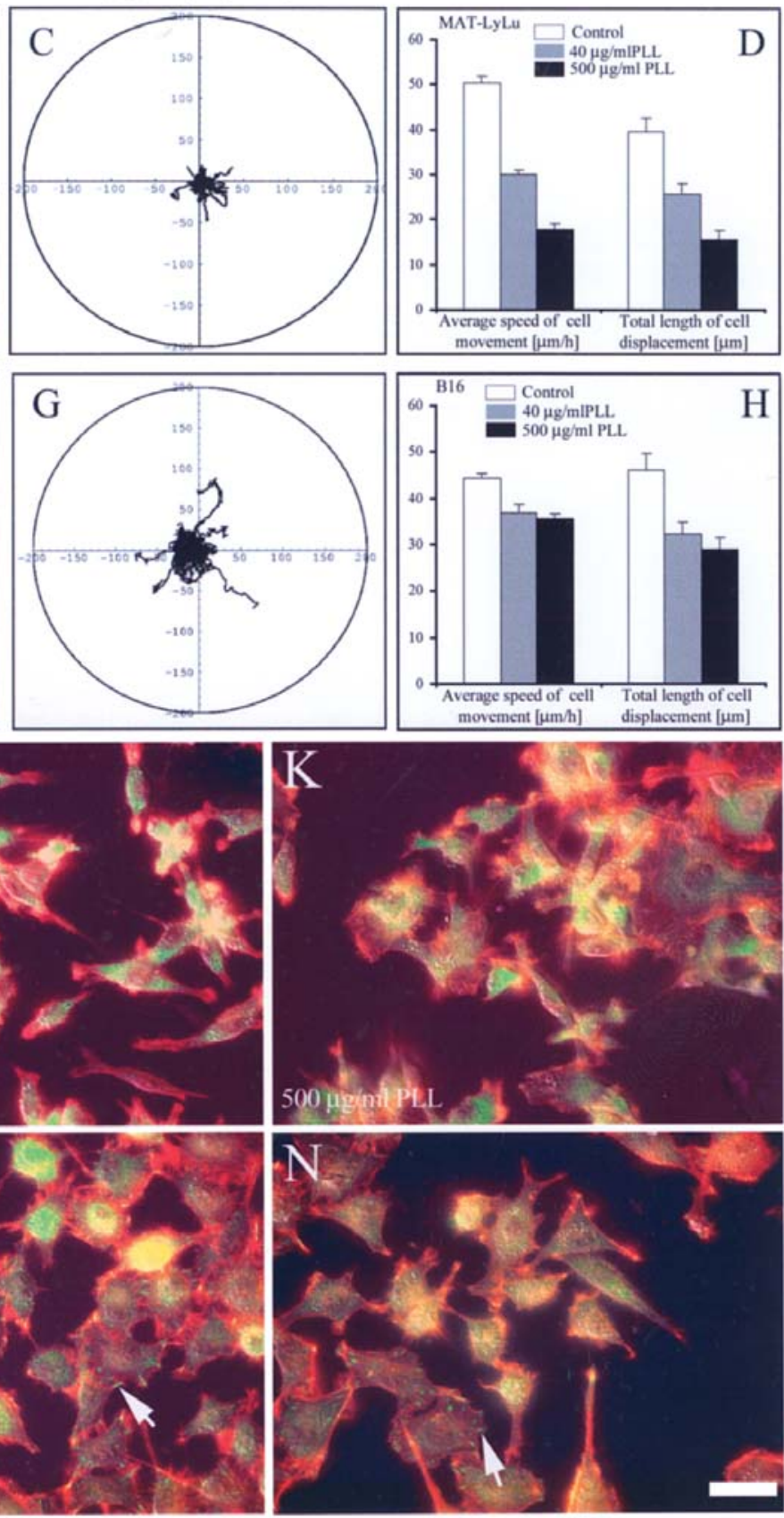

Figure 1. Effect of poly-L-lysine-coated substrata on the motility and actin cytokeleton architecture of MAT-LyLu and B16 cells. Trajectories of MAT-LyLu (A-C) and B16 (E-G) cells cultured in control conditions (A and E) and on substrata covered with 40 (B and F) and $500 \mu \mathrm{g} / \mathrm{ml}$ of poly-L-lysine (C and G) are presented in the form of circular diagrams (axis scale in $\mu \mathrm{m}$ ) drawn with the initial point of each trajectory placed at the origin of the plot. Cell trajectories were registered $24 \mathrm{~h}$ after seeding on the substrata for $4 \mathrm{~h}$ at $300 \mathrm{sec}$ time intervals. Increased adhesiveness of PLL-coated substrata reduces the rate of the displacements of MAT-LyLu and B16 cells (D and H, respectively, for statistical analysis see Table I). Decreased motile activity of the two cell lines cultured on PLL-coated substrata did not correlate with changes in the organisation of the actin cytoskeleton as visualised by F-actin (red) and vinculin (green) staining of MAT-LyLu (I-K) and B16 cells (L-N) cultured in control conditions (I and L) and on substrata covered with 40 (J and M) and $500 \mu \mathrm{g} / \mathrm{ml}$ of poly-L-lysine (K and N). Bar, $20 \mu \mathrm{m}$.

Inhibition of cancer cell motility by PLL-coated substrata enhances GJIC. MAT-LyLu cells were previously shown to express Cx43 and to display relatively high levels of GJIC (14). Analyses were performed in order to clarify whether the inhibition of MAT-LyLu cellular translocation by PLL-coated substrata affected the intracellular localisation of $\mathrm{Cx} 43$. They revealed an increase in the abundance of Cx43-specific plaques at cell-to-cell contacts in PLL-treated populations of MAT-LyLu cells (marked with arrows; Fig. 2B and C, cf. A).
Furthermore, a punctate Cx43-positive staining was observed in melanoma B16 cell populations (Fig. 2 D), where the intensity increased in B16 cells cultured on PLL-coated substrata (Fig. 2E and F) and was even more distinct than in MAT-LyLu cell cultures (Table II). Since a pronounced cytosolic expression of $\mathrm{Cx} 43$ could be seen, immunoblot analyses were performed to estimate the effect of PLL-coated substrata on $\mathrm{Cx} 43$ protein and phosphorylation levels in the two cell populations. While B16 cells displayed higher Cx43 
Table I. A summary of quantitative data showing the effect of poly-L-lysine-coated substrata on the motility of MAT-LyLu and $\mathrm{B} 16$ cells.

MAT-LyLu

Parameters $( \pm$ SEM)

Concentration of poly-L-lysine $(\mu \mathrm{g} / \mathrm{ml})$

\begin{tabular}{rrr}
\hline 0 & 40 & 500 \\
$50.4 \pm 1.4$ & $30.0 \pm 1.0^{c}$ & $17.9 \pm 1.1^{\mathrm{c}}$ \\
$39.4 \pm 3.1$ & $25.8 \pm 2.2^{\mathrm{c}}$ & $15.6 \pm 2.0^{\mathrm{c}}$ \\
$9.9 \pm 0.8$ & $6.5 \pm 0.6^{\mathrm{c}}$ & $3.9 \pm 0.5^{\mathrm{c}}$
\end{tabular}

B16

Parameters $( \pm$ SEM)

\begin{tabular}{ccc}
\multicolumn{3}{c}{ Concentration of poly-L-lysine $(\mu \mathrm{g} / \mathrm{ml})$} \\
\hline 0 & 40 & 500 \\
$44.34 \pm 1.04$ & $36.84 \pm 0.78^{\mathrm{c}}$ & $35.62 \pm 0.92^{\mathrm{c}}$ \\
$45.97 \pm 3.53$ & $32.34 \pm 2.53^{\mathrm{c}}$ & $29,02 \pm 2.45^{\mathrm{c}}$ \\
$11.45 \pm 0.88$ & $8.09 \pm 0.63^{\mathrm{c}}$ & $7.23 \pm 0.61^{\mathrm{c}}$
\end{tabular}

Average speed of cell movement $(\mu \mathrm{m} / \mathrm{h})^{\mathrm{a}}$

Total length of cell displacement $(\mu \mathrm{m})$

Average rate of cell displacement $(\mu \mathrm{m} / \mathrm{h})^{\mathrm{b}}$
$45.97 \pm 3.53$

$7.23 \pm 0.61^{\mathrm{c}}$

${ }^{a}$ Average speed of cell movement is defined as the total length of cell trajectory/time of recording $(4 \mathrm{~h}) .{ }^{\mathrm{b}}$ Average rate of cell displacement is

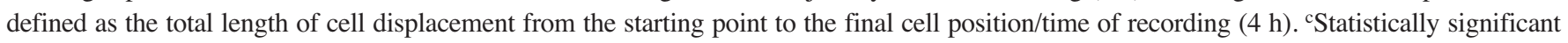
(Mann-Whitney test) probe vs control $\mathrm{p}<0.001$.

Table II. A semi-quantitative analysis of the effect of PLL-coated substrata on the abundance of Cx43-positive plaques in rat prostate MAT-LyLu and melanoma B16 cell populations, indicating the intensity of punctate Cx43-specific fluorescence.

Cancer cell line

Concentration of poly-L-lysine $(\mu \mathrm{g} / \mathrm{ml})$

\begin{tabular}{lccc} 
& 0 & 40 & 500 \\
\hline MAT-LyLu & $(+)$ & $(++)$ & $(++)$ \\
B16 & $(+)$ & $(++)$ & $(+++)$ \\
\hline
\end{tabular}

$(+)$, Control; $(++)$, considerably higher than in control; $(+++)$, high abundance.

protein levels than MAT-LyLu cells (Fig. 1G), no significant effect of PLL-coated substrata on the Cx43 protein levels in B16 cells was observed (Fig. 2G). The intensities of bands corresponding to phosphorylated $(\sim 45 \mathrm{kDa})$ and unphosphorylated ( $\sim 43 \mathrm{kDa}) \mathrm{Cx} 43$ remained similar for the cells cultured on PLL-coated and control substrata.

Subsequently, a calcein transfer analysis (23) was performed to elucidate the effect of the increased abundance of Cx43-positive plaques observed in PLL-treated cancer cell populations on GJIC intensity. Elevated calcein transfer was observed in MAT-LyLu cell populations cultured on PLLcoated culture substrata compared to control variants (Fig. 2I, $\mathrm{J}$ cf. H), represented by a significantly increased $c_{r}$ value (Fig. 2K). Furthermore, high GJIC observed for the first time in B16 cell populations (Fig. 2L) was enhanced on PLLcoated substrata when compared to control variants (Fig. 2M, $\mathrm{N}$, c.f. L, summarised in $\mathrm{O}$ ). These data indicate that the decrease in the intensity of MAT-LyLu and B16 cell intercellular translocation affects Cx43 trafficking and alleviates the formation of $\mathrm{Cx} 43$-positive plaques at cell-to-cell contacts independently of its expression and phosporylation. This leads, in consequence, to an enhancement of GJIC in cancer cell populations.

\section{Discussion}

Since functional links between GJIC and cell motility were identified, attention has been focused on the influence of connexins on cellular motile properties. For example, Cx43 was shown to affect the migration of neural crest cells during embryogenesis (24-26), while the establishment of GJIC facilitated endothelial transmigration and extravasation of leukocytes and monocytes (27). In contrast, the few studies on cancer models that were performed to date did not reveal any effect of GJIC on cancer cell motility. The restoration of GJIC in HeLa cell populations by a stable $\mathrm{Cx} 43$ transfection 

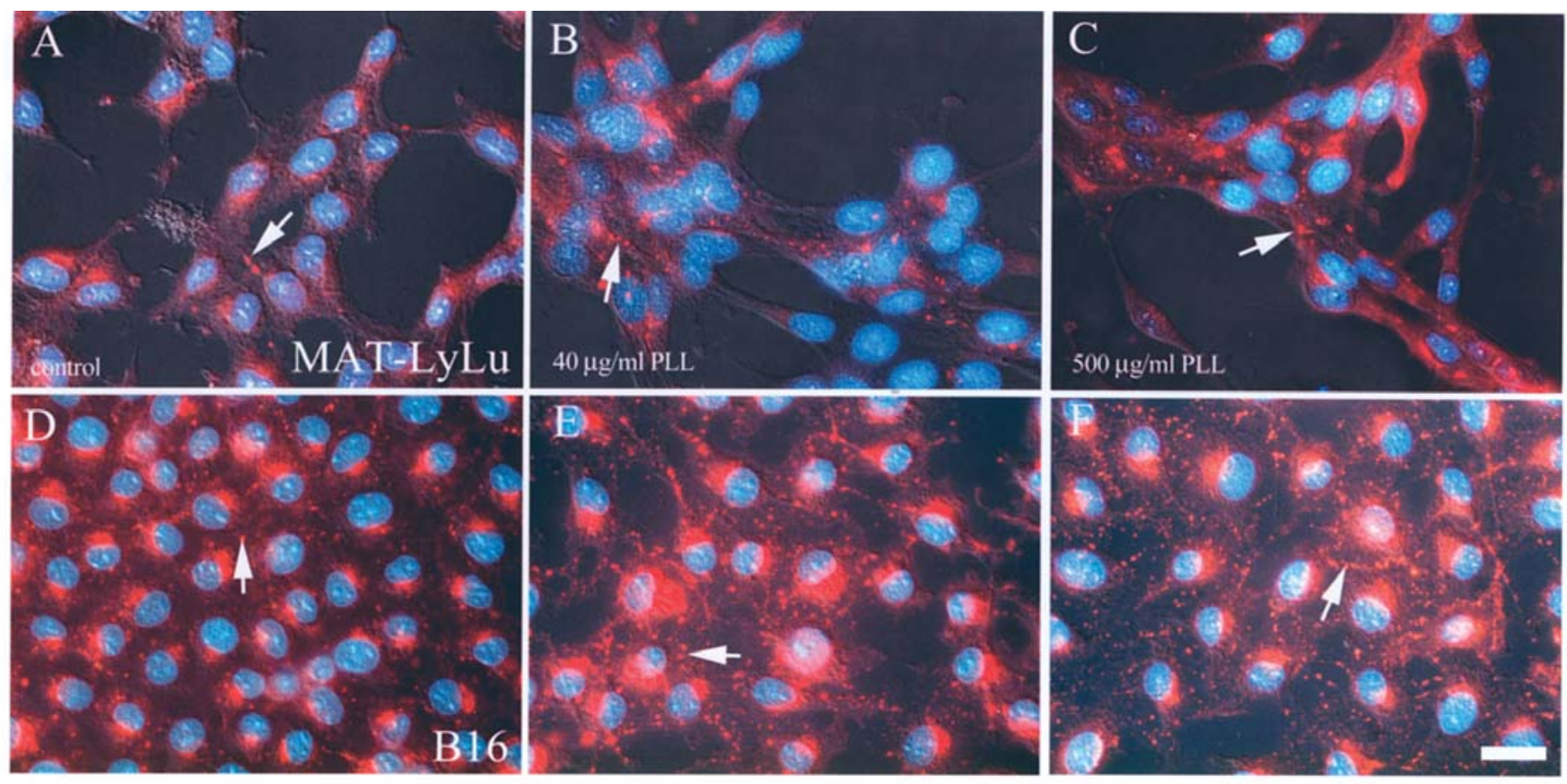

G
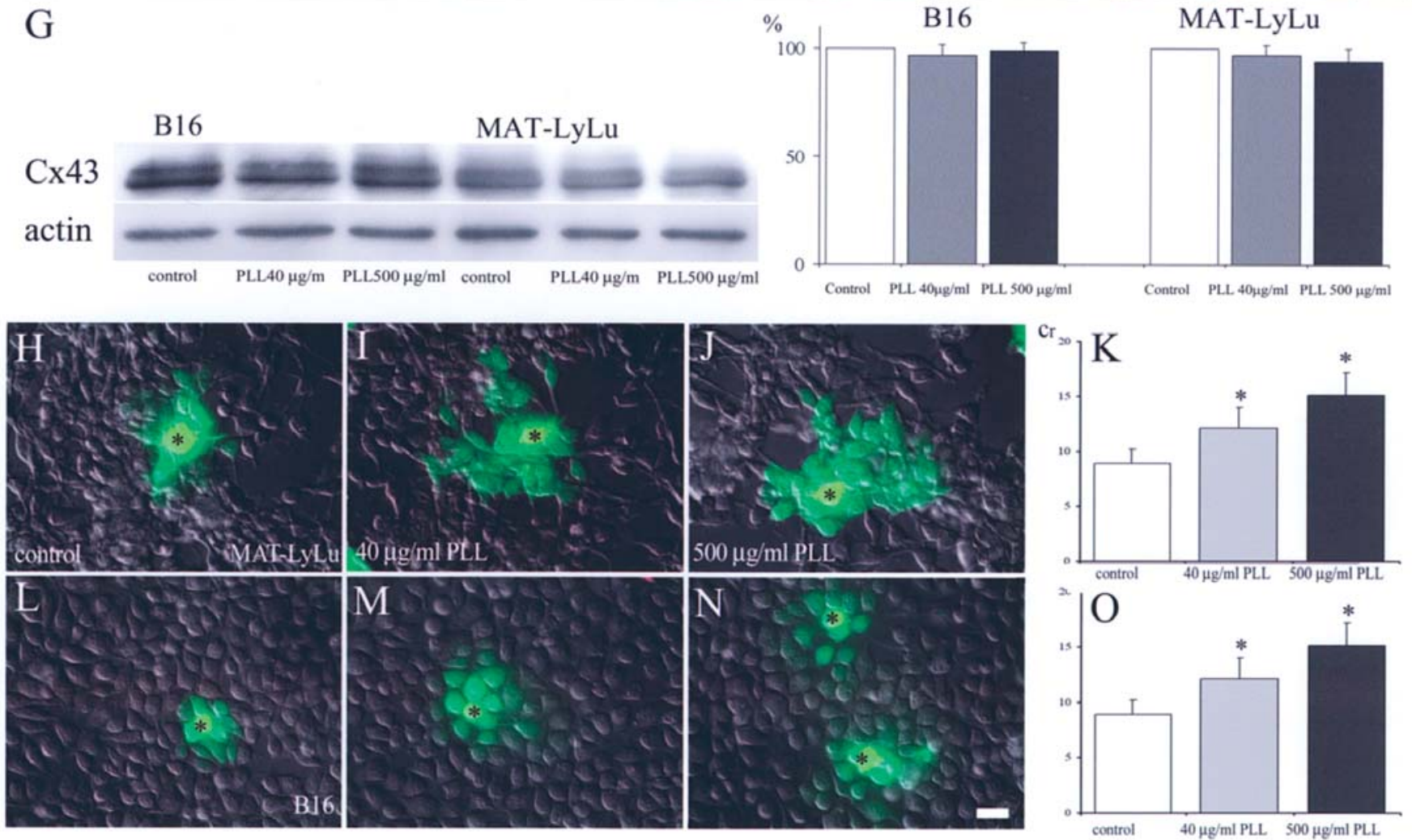

Figure 2. Effect of poly-L-lysine-coated substrata on the intracellular localisation, expression and phosphorylation of Cx43 (A-G) and GJIC intensity (H-O) in MAT-LyLu and B16 cell populations. An increase in the abundance of Cx43-positive plaques (red) was observed in cell-to-cell contacts in MAT-LyLu cell populations cultured on substrata coated with 40 (B) and $500 \mu \mathrm{g} / \mathrm{ml}$ (C) PLL compared to control conditions (A). A similar enrichment in mature Cx43-positive plaques was seen in B16 cell populations cultured on PLL-coated substrata (E,F cf. D for control). No effect of PLL-coated substrata on Cx43 expression levels was revealed by immunoblot analyses of total protein and phosphorylation levels of Cx43 in B16 and MAT-LyLu populations cultured on control and PLL-coated substrata (N=3; Fig. 2G). However, an increase in abundance of Cx43-positive plaques on PLL-coated substrata correlated with enhanced GJIC in MAT-LyLu (H-J) and B16 (L-N) cell populations, which was illustrated by the intensified transfer of calcein (green) from donor (DiI-loaded cells (yellow, marked with asterisk) placed in contact with acceptor cells cultured on the control substrata ( $\mathrm{H}$ and $\mathrm{L}$ ) and substrata covered with 40 (I and $\mathrm{M}$ ) and $500 \mu \mathrm{g} / \mathrm{ml}$ of poly-L-lysine ( $\mathrm{J}$ and $\mathrm{N}$ ). This is illustrated by $\mathrm{c}_{\mathrm{r}}$ values calculated for MAT-LyLu $(\mathrm{K})$ and B16 cells (K). A-F represent merges of images of Cx43- (red) and DNA-specific (blue) staining with Nomarski interference contrast pictures (grey). I-O depicts merges of calcein (green), DiI (red) and Nomarski interference contrast pictures. ${ }^{*} \mathrm{P}<0.05$ as determined by a paired Student's t-test. Scale bars, $20 \mu \mathrm{m}$.

exerted no detectable effect on their motile behaviour $(28,29)$. Furthermore, Dunning rat prostate carcinoma MATLyLu cells were shown to exhibit similar motile properties to their non-metastatic (AT-2) counterparts, while displaying considerably higher $\mathrm{Cx} 43$ expression levels and GJIC intensity (14). In our study, the opposite effect, i.e. the influence of cell 
motility on GJIC intensity in cancer cell populations was experimentally addressed using MAT-LyLu cells as a cellular model. The inhibition of cell motility by PLL-coated substrata led to a significant and dose-dependent increase of Cx43-mediated GJIC intensity in MAT-LyLu cell populations. A similar correlation was observed in mouse melanoma B16 cell populations, suggesting that the interrelation between cellular translocation and GJIC is not cell type-specific. Thus, using a simple experimental approach, we demonstrated that cancer cell motility affects the stability of gap junctions in cancer cell populations, in this way modulating the intensity of GJIC.

At least two lines of evidence indicate that this previously unaddressed way of interrelation between the function of connexins and cell adhesion results from the direct effect of the impairment of cancer cell motility on GJIC in analysed cell populations. PLL is known to maintain poorly adhesive cells in an adherent state $(19,20)$ and affects the cytoskeleton architecture in adhesive cells (30). The effect of impaired cell adhesion on connexin expression and function has been reported (31). However, based on our experience, PLL-coated substrata immobilized MAT-LyLu and B16 cells independently of microfilament function, since no significant effect of PLLcoated substrata on the architecture of actin cytoskeleton was observed. The stabilization of intercellular contacts by PLL-coated substrata did not affect Cx43 expression and phosphorylation levels, demonstrating that the observed phenomena are independent of the pathways regulating Cx43 function in MAT-LyLu and B16 cell populations. Since the internalisation of gap junctions takes place when a migrating cell moves along and establishes new contacts in the proximal cell region (32), a shift in the proportion between surface and cytoplasmic pools of $\mathrm{Cx} 43$ seems to account for the increase in the number of $\mathrm{Cx} 43$-positive plaques and an enhancement of GJIC in MAT-LyLu and B16 cell populations immobilised by PLL-coated substrata. This notion remains in accordance with the finding that connexins undergo a rapid intracellular turnover and connexin-positive membranous plaques are predominantly responsible for mediating GJIC (2). As mechanical stability of gap junctions depends on cell adhesion molecules, their lack at cell-to-cell contacts observed in analysed cell populations (not shown) can further sensitize GJ channels to cell motility.

The observed correlation between the impairment of cancer cell motility and enhanced GJIC may have strong implications for the interpretation of the data concerning the involvement of the two parameters in carcinogenesis. In principle, we demonstrated that 'contact-stimulation' of MAT-LyLu and B16 cell movements $(15,16)$ attenuates GJIC in the two cancer cell populations. The disturbance of GJIC participates in cancer development (7). Therefore, this previously unaddressed correlation between the inhibition of 'contactstimulated' cell movement and an enhancement of GJIC is another element of the network of interactions between cancer cell motility and GJIC $(12,26)$. As a consequence, mechanisms of an enhancement of GJIC in cell populations subjected to para-pharmaceutical agents should be reconsidered as at least in part resulting from the stabilisation of gap junctions in populations of immobilised cells (33).
In summary, we demonstrated that tumor cell motile behaviour seems crucial for the function of gap junctions, implying the necessity for the inclusion of cell motility as a factor determining gap junctional communication. While further study is needed to define reciprocal interrelations between cell motility, intercellular communication and cell adhesion during tumor development, the data presented here expand our understanding of the role of tumor cell motility in modulating cellular functions involved in tumorigenesis.

\section{Acknowledgements}

We thank Professor Wlodzimierz Korohoda for his continuous support and helpful discussions and Ewa Niedzialkowska for her excellent technical support. This study was supported by grants PB 2P04C 12529 and PB 2P04C 00828 from the Ministry of Scientific Research and Higher Education.

\section{References}

1. Sohl G and Willecke K: Gap junctions and the connexin protein family. Cardiovasc Res 62: 228-232, 2004.

2. Rutz ML and Hulser DF: Supramolecular dynamics of gap junctions. Eur J Cell Biol 80: 20-30, 2001.

3. Harris AL: Emerging issues of connexin channels: biophysics fills the gap. Q Rev Biophys 34: 325-472, 2001

4. Fujimoto K, Nagafuchi A, Tsukita S, et al: Dynamics of connexins, E-cadherin and alpha-catenin on cell membranes during gap junction formation. J Cell Sci 110: 311-322, 1997.

5. Zuppinger C, Schaub MC and Eppenberger HM: Dynamics of early contact formation in cultured adult rat cardiomyocytes studied by N-cadherin fused to green fluorescent protein. J Mol Cell Cardiol 32: 539-555, 2000.

6. Wei CJ, Xu X and Lo CW: Connexins and cell signaling in development and disease. Annu Rev Cell Dev Biol 20: 811-838, 2004

7. Yamasaki H, Krutovskikh V, Mesnil M, et al: Role of connexin (gap junction) genes in cell growth control and carcinogenesis. C R Acad Sci III 322: 151-159, 1999.

8. Czyz J: Stage-specific function of gap junctions during tumorigenesis. Cell Mol Biol Lett 13: 92-102, 2008.

9. Moorby $\mathrm{C}$ and Patel M: Dual functions for connexins: Cx43 regulates growth independently of gap junction formation. Exp Cell Res 271: 238-248, 2001.

10. Qin H, Shao Q, Curtis H, et al: Retroviral delivery of connexin genes to human breast tumor cells inhibits in vivo tumor growth by a mechanism that is independent of significant gap junctional intercellular communication. J Biol Chem 277: 29132-29138, 2002.

11. Zhang YW, Nakayama K, Nakayama K and Morita I: A novel route for connexin 43 to inhibit cell proliferation: negative regulation of S-phase kinase-associated protein ( $\mathrm{Skp} 2)$. Cancer Res 63: 1623-1630, 2003.

12. Lin JH, Takano T, Cotrina ML, et al: Connexin 43 enhances the adhesivity and mediates the invasion of malignant glioma cells. J Neurosci 22: 4302-4311, 2002.

13. Gupta GP and Massague J: Cancer metastasis: building a framework. Cell 127: 679-695, 2006.

14. Miekus K, Czernik M, Sroka J, Czyz J and Madeja Z: Contact stimulation of prostate cancer cell migration: the role of gap junctional coupling and migration stimulated by heterotypic cell-to-cell contacts in determination of the metastatic phenotype of Dunning rat prostate cancer cells. Biol Cell 97: 893-903, 2005.

15. Madeja Z, Miekus K, Sroka J, Djamgoz MB and Korohoda W: Homotypic cell-cell contacts stimulate the motile activity of rat prostate cancer cells. BJU Int 88: 776-786, 2001.

16. Madeja Z, Szymkiewicz I, Zaczek A, et al: Contact-activated migration of melanoma B16 and sarcoma XC cells. Biochem Cell Biol 79: 425-440, 2001 .

17. Jessel N, Oulad-Abdelghani M, Meyer F, et al: Multiple and time-scheduled in situ DNA delivery mediated by betacyclodextrin embedded in a polyelectrolyte multilayer. Proc Natl Acad Sci USA 103: 8618-8621, 2006. 
18. Wang GX, Deng XY, Tang CJ, et al: The adhesive properties of endothelial cells on endovascular stent coated by substrates of poly-l-lysine and fibronectin. Artif Cells Blood Substit Immobil Biotechnol 34: 11-25, 2006.

19. Stepien E, Stanisz J and Korohoda W: Contact guidance of chick embryo neurons on single scratches in glass and on underlying aligned human skin fibroblasts. Cell Biol Int 23: 105-116, 1999.

20. Mokry J and Karbanova J: Foetal mouse neural stem cells give rise to ependymal cells in vitro. Folia Biol (Praha) 52: 149-155, 2006.

21. Sroka J, Antosik A, Czyz J, et al: Overexpression of thioredoxin reductase 1 inhibits migration of HEK-293 cells. Biol Cell 99: 677-687, 2007.

22. Czyz J, Guan K, Zeng Q and Wobus AM: Loss of beta 1 integrin function results in upregulation of connexin expression in embryonic stem cell-derived cardiomyocytes. Int J Dev Biol 49: 33-41, 2005.

23. Czyz J, Irmer U, Schulz G, Mindermann A and Hulser DF: Gapjunctional coupling measured by flow cytometry. Exp Cell Res 255: 40-46, 2000.

24. Huang GY, Cooper ES, Waldo K, et al: Gap junction-mediated cell-cell communication modulates mouse neural crest migration. J Cell Biol 143: 1725-1734, 1998.

25. Xu X, Li WE, Huang GY, et al: Modulation of mouse neural crest cell motility by $\mathrm{N}$-cadherin and connexin 43 gap junctions. J Cell Biol 154: 217-230, 2001.

26. Elias LA, Wang DD and Kriegstein AR: Gap junction adhesion is necessary for radial migration in the neocortex. Nature 448: 901-907, 2007.
27. Eugenin EA, Branes MC, Berman JW and Saez JC: TNF-alpha plus IFN-gamma induce connexin 43 expression and formation of gap junctions between human monocytes/macrophages that enhance physiological responses. J Immunol 170: 1320-1328, 2003.

28. Graeber SH and Hulser DF: Connexin transfection induces invasive properties in HeLa cells. Exp Cell Res 243: 142-149, 1998.

29. Czyz J, Madeja Z, Irmer U, Korohoda W and Hulser DF: Flavonoid apigenin inhibits motility and invasiveness of carcinoma cells in vitro. Int J Cancer 114: 12-18, 2005.

30. Cordes $\mathrm{N}$ and van Beuningen D: Cell adhesion to the extracellular matrix protein fibronectin modulates radiation-dependent $\mathrm{G} 2$ phase arrest involving integrin-linked kinase (ILK) and glycogen synthase kinase-3beta (GSK-3beta) in vitro. Br J Cancer 88: 1470-1479, 2003.

31. El Sabban ME, Sfeir AJ, Daher MH, et al: ECM-induced gap junctional communication enhances mammary epithelial cell differentiation. J Cell Sci 116: 3531-3541, 2003.

32. Jordan K, Chodock R, Hand AR and Laird DW: The origin of annular junctions: a mechanism of gap junction internalization. J Cell Sci 114: 763-773, 2001.

33. Czernik M, Sroka J, Madeja Z, Czyz J: Apigenin inhibits growth and motility but increases gap junctional coupling intensity in rat prostate carcinboma (MAT-LYLu) cell populations. Cell Mol Biol Lett (In press). 